\title{
Protective effects of bilberry and lingonberry extracts against blue light-emitting diode light-induced retinal photoreceptor cell damage in vitro
}

Kenjirou Ogawa', Yoshiki Kuse', Kazuhiro Tsuruma', Saori Kobayashi², Masamitsu Shimazawa' and Hideaki Hara ${ }^{1 *}$

\begin{abstract}
Background: Blue light is a high-energy or short-wavelength visible light, which induces retinal diseases such as age-related macular degeneration and retinitis pigmentosa. Bilberry (Vaccinium myrtillus L.) and lingonberry (Vaccinium vitis-idaea) contain high amounts of polyphenols (anthocyanins, resveratrol, and proanthocyanidins) and thus confer health benefits. This study aimed to determine the protective effects and mechanism of action of bilberry extract (B-ext) and lingonberry extract (L-ext) and their active components against blue light-emitting diode (LED) light-induced retinal photoreceptor cell damage.
\end{abstract}

Methods: Cultured murine photoreceptor $(661 \mathrm{~W}$ ) cells were exposed to blue LED light following treatment with B-ext, L-ext, or their constituents (cyanidin, delphinidin, malvidin, trans-resveratrol, and procyanidin B2). $661 \mathrm{~W}$ cell viability was assessed using a tetrazolium salt (WST-8) assay and Hoechst 33342 nuclear staining, and intracellular reactive oxygen species (ROS) production was determined using $\mathrm{CM}-\mathrm{H}_{2} \mathrm{DCFDA}$ after blue LED light exposure. Activation of p38 mitogen-activated protein kinase (p38 MAPK), nuclear factor-kappa B (NF-kB), and LC3, an ubiquitin-like protein that is necessary for the formation of autophagosomes, were analyzed using Western blotting. Caspase-3/7 activation caused by blue LED light exposure in $661 \mathrm{~W}$ cells was determined using a caspase-3/7 assay kit.

Results: B-ext, L-ext, NAC, and their active components improved the viability of $661 \mathrm{~W}$ cells and inhibited the generation of intracellular ROS induced by blue LED light irradiation. Furthermore, B-ext and L-ext inhibited the activation of p38 MAPK and NF-KB induced by blue LED light exposure. Finally, B-ext, L-ext, and NAC inhibited caspase-3/7 activation and autophagy.

Conclusions: These findings suggest that B-ext and L-ext containing high amounts of polyphenols exert protective effects against blue LED light-induced retinal photoreceptor cell damage mainly through inhibition of ROS production and activation of pro-apoptotic proteins.

Keywords: Anthocyanin, Bilberry, Blue LED light, Lingonberry, Proanthocyanidin, Resveratrol, Retinal photoreceptor

\footnotetext{
* Correspondence: hidehara@gifu-pu.ac.jp

'Molecular Pharmacology, Department of Biofunctional Evaluation, Gifu

Pharmaceutical University, 1-25-4 Daigaku-nishi, Gifu 501-1196, Japan

Full list of author information is available at the end of the article
} 


\section{Background}

High-energy visible light has a wavelength in the range of 380 to $530 \mathrm{~nm}$ and is present in sunlight, fluorescent light, and light-emitting diode (LED) light. Blue light (from 450 to $495 \mathrm{~nm}$ ) is high-energy visible light and is related to the pathogenesis of age-related macular degeneration and retinitis pigmentosa [1,2]. A previous report suggested that retinal damage is inversely proportional to wavelength (from 379 to $559 \mathrm{~nm}$ ) of light in a rat in vivo model [3]. In another previous study using rhesus macaque, retinal dysfunction and damage induced by blue LED light were observed as residual infiltration in retinal pigment endothelial (RPE) cells and the photoreceptor outer segment [4]. Blue light-induced RPE cell damage is caused by the accumulation of lipofuscin, such as bis-retinoid, $N$-retinylidene- $N$-retinylethanolamine (A2E), and its photoisomers, which lead to reactive oxygen species (ROS) generation by blue light stimulation in the mitochondria, resulting in membrane lipid peroxidation [5]. RPE cell death induced by blue light is mediated by the activation of caspase-3; therefore, nuclear apoptosis is attenuated by the caspase-3 inhibitor Z-DEVD-fmk [6]. Blue lightinduced retinal photoreceptor cell damage is also mediated by caspase-3 [7] and rhodopsin in vivo, and the extent of its bleaching and its regeneration and visual transduction proteins determine the degree of damage [8].

Bilberry (Vaccinium myrtillus L.) and lingonberry (Vaccinium vitis-idaea), members of the Ericaceous family, grow in the forests of northern Europe. Bilberry contains 15 different anthocyanins, including 5 anthocyanidins (delphinidin, cyanidin, malvidin, petunidin, and peonidin), and 3 sugars (glucose, galactose, and arabinose). The bilberry extract (B-ext) has potent antioxidant properties [9]; inhibits platelet aggregation [10]; and improves vascular tone, blood flow, and vasoprotection [11,12]. Furthermore, bilberry has been reported to improve visual function in animal models and clinical trials [13,14]. Animal studies have demonstrated B-ext to be beneficial in preventing retinal inflammation and cataracts [15]. Our previous studies have shown a neuroprotective effect against retinal neuronal damage induced by $N$-methyl-D-aspartic acid in mice [16] and an inhibitory effect against angiogenesis in a mouse model of oxygen-induced retinopathy [17]. Lingonberry is used in traditional medicine for the treatment of frequent urination, sore eyes, toothache, snow blindness, and thrush [18].

Lingonberry extract (L-ext) contains high amounts of phenolic antioxidants (trans-resveratrol and proanthocyanidin). We previously reported that B-ext, L-ext, and their active components (delphinidin, cyanidin, malvidin, resveratrol, procyanidin) protected against retinal photoreceptor cell damage induced by ultraviolet A light (wavelength of $365 \mathrm{~nm}$ ) exposure [19]. However, the blue light has a high permeability, higher damage, and risk to eye than ultraviolet light, and preventing blue light-induced damage is very important. Thus, the present study aimed to investigate the effects of B-ext, L-ext and their active components, and to elucidate their mechanism of action against blue light (high specificity blue light by LED)-induced retinal photoreceptor cell damage in vitro. This study demonstrated that bilberry and lingonberry containing anthocyanidins, procyanidin, and resveratrol exert protective effects against blue LED light-induced retinal photoreceptor cell damage by regulating the activation of NF- $\mathrm{KB}, \mathrm{p} 38$ MAPK, autophagy, and caspase-3/7 mainly through suppression of ROS generation.

\section{Methods}

\section{Materials}

B-ext (bilberry ethanol extract containing anthocyanins) and L-ext (lingonberry ethanol extract containing transresveratrol and proanthocyanidins) were purchased from Beijing Gingko Group Japan Co. Ltd. (Tokyo, Japan), and their compositions were confirmed by high performance liquid chromatography (HPLC). Previously, the active components of both extracts were analyzed by HPLC and UV-visible absorption spectroscopy with each standard preparation [19]. B-ext contained $38.4 \%$ anthocyanins, $14.1 \%$ delphinidin, $9.1 \%$ cyanidin, and $6.1 \%$ malvidin. L-ext contained $10.6 \%$ trans-resveratrol and $43.0 \%$ proanthocyanidin. The rest of the composition in both extracts was mostly carbohydrates. Delphinidin, cyanidin, malvidin, and procyanidin B2 were purchased from Extrasynthese (Genay Cedex, France). trans-Resveratrol was purchased from Tokyo Chemical Industry Co. Ltd. (Tokyo, Japan). N-Acetyl-L-cysteine (NAC), a positive control antioxidant, was purchased from SigmaAldrich (St Louis, MO, USA). Cell Counting Kit-8 (CCK8) was purchased from Dojindo Laboratories (Kumamoto, Japan). Hoechst 33342, propidium iodide (PI), and 5-(and6)-chloromethyl-2,7- dichlorodihydrofluorescein diacetate acetyl ester (CM- $\mathrm{H}_{2}$ DCFDA) were purchased from Invitrogen (Eugene, OR, USA). Antibodies against phosphorylated p38 MAPK, total p38 MAPK, activated NF-kB, total NF- $\mathrm{kB}$, and LC3 were purchased from Cell Signaling Technology (Beverly, MA, USA). Antibody against $\beta$-actin was purchased from Sigma-Aldrich. The Caspase-Glo ${ }^{\circ}$ 3/7 assay kit was purchased from Promega Co. (Madison, Wisconsin, USA). The $661 \mathrm{~W}$ cells were a kind gift from Dr. Muayyad R. Al-Ubaidi (University of Oklahoma Health Sciences Center, Oklahoma City, OK, USA). The blue LED light irradiation devices with 12 blue LED bulbs (wavelength of 460-470 nm) were purchased from M-trust Co. Ltd. (Hyogo, Japan). The LM-332 light meter was purchased from AS ONE Co. Ltd. (Osaka, Japan). 


\section{Cell culture}

The $661 \mathrm{~W}$ cells were maintained in Dulbecco's modified Eagle's medium (DMEM) containing 10\% fetal bovine serum (FBS), $100 \mathrm{U} / \mathrm{mL}$ penicillin, and $100 \mu \mathrm{g} / \mathrm{mL}$ streptomycin at $37^{\circ} \mathrm{C}$ in a humidified atmosphere with $5 \% \mathrm{CO}_{2}$. Cells were passaged by trypsinization every $2-3 \mathrm{~d}$.

\section{Measurement of cellular metabolic activity after blue LED light exposure}

$661 \mathrm{~W}$ cells $\left(3 \times 10^{3}\right.$ cells $\left./ 100 \mu \mathrm{L}\right)$ were seeded onto a 96-well plate and cultured at $37^{\circ} \mathrm{C}$ for $24 \mathrm{~h}$. We first investigated the effects of NAC, bilberry, lingonberry, and their active components at various concentrations and determined the effective extract concentrations. In this study, we presented the data on specific extract concentrations of each sample against blue LED lightinduced photoreceptor cell damage. At 70-80\% confluence, the medium was replaced by DMEM containing $1 \% \mathrm{FBS}$ and was placed at $37^{\circ} \mathrm{C}$ for $3 \mathrm{~h}$. Then, $1 \%$ FBSDMEM containing 9-cis-retinal (at a final concentration of $2.5 \mu \mathrm{M})$ was added to all wells. After $4 \mathrm{~h}$ of treatment with 9-cis-retinal, 1\% FBS-DMEM containing B-ext (at final concentrations of $1-10 \mu \mathrm{g} / \mathrm{mL}$ ), L-ext (at final concentrations of $1-10 \mu \mathrm{g} / \mathrm{mL}$ ), NAC (at final concentrations of 0.3 and $1 \mu \mathrm{M}$ ), anthocyanidins (at final concentrations of 1-10 $\mu \mathrm{M}$ ), trans-resveratrol (at final concentrations of $1-10 \mu \mathrm{M}$ ), or procyanidin B2 (at final concentrations of $1-10 \mu \mathrm{M}$ ) was added to each well. The cells treated with DMEM containing only 1\% FBS and exposed to blue LED light were designated as the vehicle group and used for comparison with the groups treated with each agent. After $1 \mathrm{~h}$ of preincubation with agents, the $661 \mathrm{~W}$ cells were exposed to $2500 \mathrm{~lx}$ of blue LED light at wavelength $460-470 \mathrm{~nm}$ for $6 \mathrm{~h}$ (Figure 1A and $\mathrm{B})$; the cellular metabolic activity was then immediately measured by using a water-soluble tetrazolium salt,2-(2-methoxy-4-nitrophenyl)-3-(4-nitrophenyl)-5-
(2,4-disulfophenyl)-2H-tetrazolium monosodium salt (WST-8). Briefly, $10 \mu \mathrm{L}$ of CCK-8 was added to each well, and the cells were incubated at $37^{\circ} \mathrm{C}$ for $2 \mathrm{~h}$; the absorbance was measured at $492 \mathrm{~nm}$ (reference wavelength, $660 \mathrm{~nm}$ ) using SkanIt Re for Varioskan Flash 2.4 (ThermoFisher Scientific Inc., Waltham, MA, USA).

\section{Cell death analysis after blue LED light exposure}

After $12 \mathrm{~h}$ of blue LED light exposure, Hoechst 33342 (excitation, $360 \mathrm{~nm}$; emission, $490 \mathrm{~nm}$ ) and PI were added to the culture medium at final concentrations of 8.1 and $1.5 \mu \mathrm{M}$, respectively, followed by incubation for $15 \mathrm{~min}$. Micrographs through fluorescence filters for Hoechst 33342 (U-MWU, Olympus Co., Tokyo, Japan) and PI (U-MWIG, Olympus) were acquired using a chargecoupled device camera (DP30BW, Olympus). The number of dead cells was determined.

\section{Measurement of cellular ROS production after blue light exposure}

Intracellular ROS production caused by blue LED light exposure in $661 \mathrm{~W}$ cells was determined using CM$\mathrm{H}_{2}$ DCFDA. CM- $\mathrm{H}_{2}$ DCFDA is converted by an intracellular esterase into dichlorodihydrofluorescein (DCFH). Then, the ROS oxidize a non-fluorescent DCFH to a fluorescent DCFH. After blue LED light exposure, $\mathrm{CM}-\mathrm{H}_{2}$ DCFDA was added to the culture medium at a final concentration of $10 \mu \mathrm{M}$, followed by incubation at $37^{\circ} \mathrm{C}$ for $1 \mathrm{~h}$. Fluorescence was then measured using a fluorescence spectrophotometer at $488 \mathrm{~nm}$ (excitation) and $525 \mathrm{~nm}$ (emission). The number of cells was determined by Hoechst 33342 staining and was used to calculate ROS production per cell [20].

\section{Western blot analysis}

The $661 \mathrm{~W}$ cells $\left(3 \times 10^{4}\right.$ cells $\left./ \mathrm{mL}\right)$ were seeded onto a 12 -well plate and cultured at $37^{\circ} \mathrm{C}$ for $24 \mathrm{~h}$. After blue

\section{A}

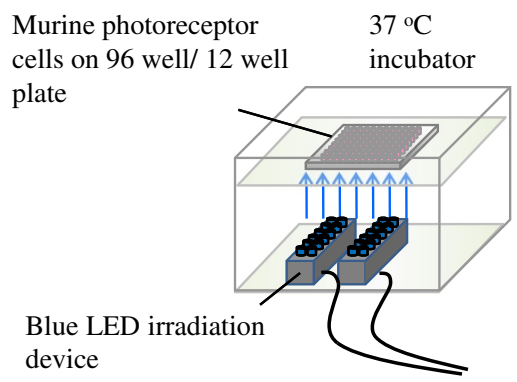

B

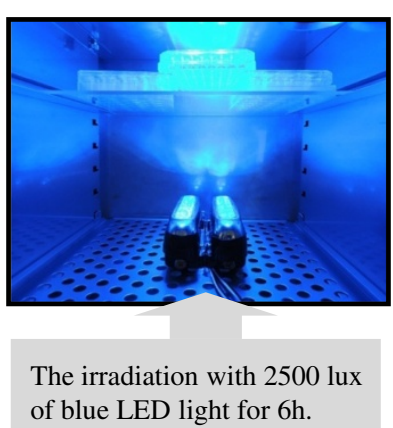

Figure 1 Model of the blue LED light irradiation-induced murine photoreceptor (661 W) cell damage. (A) 661 W cells on a 96- or 12-well plate were exposed to blue LED light irradiation at the bottom of a $37^{\circ} \mathrm{C}$ incubator. (B) A magnified view of the Blue LED light irradiation with $2500 \mathrm{~lx}$ at the bottom. 
LED light exposure, the $661 \mathrm{~W}$ cells were lysed in a cell lysis buffer (RIPA buffer) with phosphatase inhibitor cocktails 2 and 3 (P5726 and P0044, Sigma-Aldrich) and a protease inhibitor (P8340, Sigma-Aldrich). The lysate was centrifuged at $12,000 \mathrm{~g}$ for $20 \mathrm{~min}$, and the supernatant was collected for analysis. Protein concentration was determined with a BCA protein assay kit (ThermoFisher Scientific Inc.), with bovine serum albumin as standard. An equal volume of protein sample and sample buffer with 10\% 2-mercaptoethanol was electrophoresed with a $10 \%$ sodium dodecyl sulfate-polyacrylamide gel, and the separated proteins were then transferred onto a polyvinylidene difluoride membrane (Immobilon-P, Millipore Corporation, Billerica, MA, USA). The membrane was immunoblotted with the following primary antibodies: rabbit anti-phospho-p38 MAPK, rabbit anti-p38 MAPK, rabbit anti-activated NF-kB, rabbit anti-NF-kB, and rabbit anti-LC3 (1 : 1000; Cell Signaling Technology), and mouse anti- $\beta$-actin ( 1 : 5000; SigmaAldrich)*. HRP-conjugated goat anti-rabbit or goat anti-mouse secondary antibody was used (1:2000; ThermoFisher Scientific Inc.). Immunoreactive bands were visualized using a chemiluminescent substrate (ImmunoStar ${ }^{\bullet}$ LD, Wako-Junyaku Inc., Osaka, Japan). Band densities were measured using an imaging analyzer (LAS-4000 mini, Fujifilm, Tokyo, Japan), a gel analysis software (Image Reader LAS-4000, Fujifilm), and a detected band analysis software (Malti Gauge, Fujifilm).

\section{Measurement of caspase-3/7 activity after blue light exposure}

Caspase-3/7 activation caused by blue LED light exposure in $661 \mathrm{~W}$ cells was determined using a caspase-3/7 assay kit. After $12 \mathrm{~h}$ of blue LED light exposure, Caspase-Glo ${ }^{\circ}$ $3 / 7$ reagent was added to a 96-well plate, which was incubated at $37^{\circ} \mathrm{C}$ for $1 \mathrm{~h}$. The 96 -well plate was placed in a plate holder in a fluorescence spectrophotometer, and luminescence and fluorescence were measured. The number of cells was determined by Hoechst 33342 staining and used to calculate caspase-3/7 activity per cell.

\section{Statistical analysis}

Data are presented as means \pm SEM. Statistical comparisons were made using one-way analysis of variance followed by Student's $t$-test or Dunnett's multiple comparison test. A value of $p<0.05$ was considered statistically significant.

\section{Results}

Inhibitory effects of B-ext and L-ext on photoreceptor cellular morphological alterations and metabolic activity reduction after blue LED light exposure

We first established the blue LED light-induced murine photoreceptor $(661 \mathrm{~W})$ cell damage model in vitro
(Figure 1). Then, we investigated the effects of B-ext, L-ext, and NAC, a positive control antioxidant, at various concentrations, and we determined the effective concentration of each agent. We analyzed the effects of B-ext, L-ext, and NAC on the morphological alterations induced by blue LED light in the $661 \mathrm{~W}$ cell cultures. The adherence to 96-well plate bottom of vehicle-treated photoreceptor cells were attenuated and the cytomorphology of that deformed spherical, which indicated apoptotic cells, after blue LED light exposure. However, pretreatment with $10 \mu \mathrm{g} / \mathrm{mL}$ B-ext, $10 \mu \mathrm{g} / \mathrm{mL} \mathrm{L}-$ ext, or $1 \mathrm{mM}$ NAC prevented the apoptotic morphological defects by blue LED light exposure (Figure 2A). Treatment with $10 \mu \mathrm{g} / \mathrm{mL}$ B-ext, 3 and $10 \mu \mathrm{g} / \mathrm{mL}$ L-ext, or 0.3 and $1 \mathrm{mM}$ NAC significantly improved the reduction in metabolic activity of $661 \mathrm{~W}$ cells induced by blue LED light exposure (Figure 2B-D). Furthermore, B-ext and L-ext active components-delphinidin $(10 \mu \mathrm{M})$, cyanidin $(10 \mu \mathrm{M})$, malvidin $(10 \mu \mathrm{M})$, procyanidin B2 $(10 \mu \mathrm{M})$, and trans-resveratrol (3 and $10 \mu \mathrm{M})$ - significantly improved the metabolic activity of $661 \mathrm{~W}$ cells after blue LED light exposure (Figure 2E and F).

\section{Inhibitory effects of B-ext and L-ext and their active components on blue LED light-induced photoreceptor cell death}

We counted the number of blue LED light-induced dead photoreceptor cells exhibiting PI fluorescence, expressed as a percentage of cells exhibiting Hoechst 33342 fluorescence to investigate the protective effects of B-ext, L-ext, and their active components at various concentrations against blue LED light-induced cell death in 661 W cells. Representative images of Hoechst 33342 and PI staining shown in Figure 3A. Treatment with $1 \mathrm{mM}$ NAC significantly inhibited $661 \mathrm{~W}$ cell death induced by blue LED light exposure (Figure 3D). Treatment with $10 \mu \mathrm{g} / \mathrm{mL}$ B-ext, $10 \mu \mathrm{g} / \mathrm{mL}$ L-ext, B-ext active components (10 $\mu \mathrm{M}$ delphinidin, 3 and $10 \mu \mathrm{M}$ cyanidin, and $10 \mu \mathrm{M}$ malvidin), and those of L-ext (1-10 $\mu \mathrm{M}$ trans-resveratrol and 1-10 $\mu \mathrm{M}$ procyanidin B2) significantly inhibited $661 \mathrm{~W}$ cell death in a concentration-dependent manner (Figure 3B, C, E, F).

\section{Inhibitory effects of B-ext and L-ext against blue light-induced ROS production in retinal photoreceptor cell cultures}

Intercellular ROS production, which is converted to a fluorescent product $\left(\mathrm{CM}-\mathrm{H}_{2} \mathrm{DCF}\right)$ upon exposure to ROS, was increased by blue LED light exposure, and 0.3 and $1 \mathrm{mM}$ NAC significantly reduced the blue LED lightinduced ROS production in $661 \mathrm{~W}$ cells (Figure 4C). Treatment with $10 \mu \mathrm{g} / \mathrm{mL}$ B-ext, 1-10 $\mu \mathrm{g} / \mathrm{mL} \mathrm{L-ext,} \mathrm{and}$ the active components of B-ext and L-ext-delphinidin (3 and $10 \mu \mathrm{M}$ ), cyanidin (3 and $10 \mu \mathrm{M}$ ), malvidin 
A

Blue LED exposure

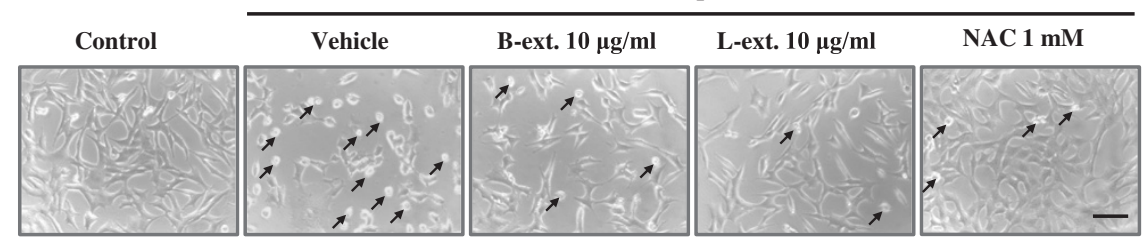

B

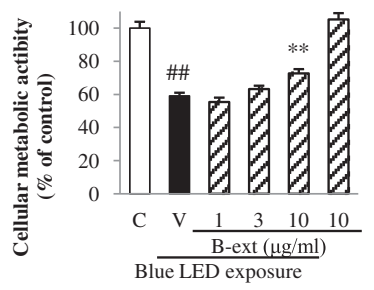

$\mathbf{E}$

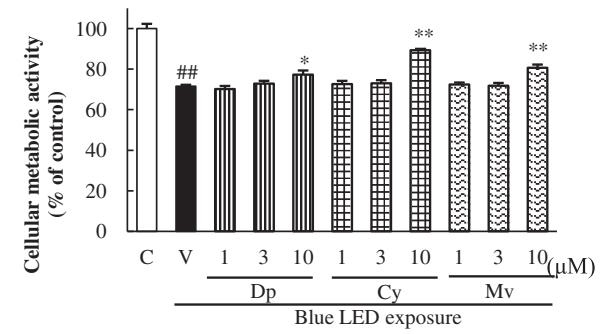

C

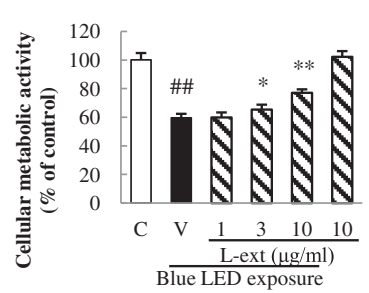

D

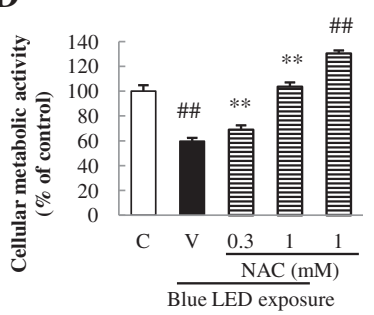

F

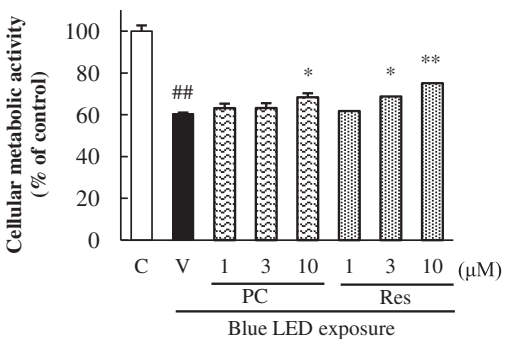

Figure 2 B-ext, L-ext, their active components, and NAC improved metabolic activity reduction in 661 W cells. (A) Cytomorphology of $661 \mathrm{~W}$ cells after $24 \mathrm{~h}$ of blue LED light exposure. Small arrows indicate apoptotic cells. Scale bar, 50 um. Inhibitory effects of B-ext (B), L-ext (C), NAC (D), delphinidin, cyanidin, malvidin (E), resveratrol, and procyanidin B2 (F) on blue LED light-induced reduction of metabolic activity in $661 \mathrm{~W}$ cells. Metabolic activity was assessed by incubating the cells with CCK-8 reagent for $2 \mathrm{~h}$ at $37^{\circ} \mathrm{C}$; photometric data were obtained at $492 / 660 \mathrm{~nm}$. Cells were treated with B-ext, L-ext, NAC, delphinidin, cyanidin, malvidin, trans-resveratrol, and procyanidin B2 for $1 \mathrm{~h}$, and then exposed to $2500 \mathrm{~lx}$ of blue LED light for $6 \mathrm{~h}$. Data are means \pm SEM $(n=6)$. C, control; $V$, vehicle; B-ext, bilberry extract; L-ext, lingonberry extract; NAC, N-acetyl-L-cysteine; Dp, delphinidin; Cy, cyanidin; Mv, malvidin; PC, procyanidin B2; Res, trans-resveratrol. ${ }^{\# \#} p<0.01$ vs. control; * $p<0.05$, ${ }^{* *} p<0.01$ vs. the vehicle-treated group (Dunnett's multiple comparison test or Student's t-test).

(10 $\mu \mathrm{M})$, trans-resveratrol (3 and $10 \mu \mathrm{M})$, and procyanidin B2 $(1-10 \mu \mathrm{M})$ - significantly reduced the blue LED light-induced ROS production in $661 \mathrm{~W}$ cells in a concentration-dependent manner (Figure 4A, B, D, E).

\section{Regulatory effects of B-ext and L-ext on the phosphorylation of stress response proteins in photoreceptor cells after blue light exposure}

We performed Western blot analysis to investigate the regulatory effects of B-ext, L-ext, and NAC on the p38 MAPK stress response pathway, NF-kB, and LC3 autophagy signaling after exposure to blue LED light. Blue LED light exposure increased the phosphorylation of p38 MAPK and NF- $\mathrm{kB}$ activation, and treatments with $10 \mu \mathrm{g} / \mathrm{mL}$ B-ext and $10 \mu \mathrm{g} / \mathrm{mL}$ L-ext significantly inhibited the activation of $\mathrm{p} 38 \mathrm{MAPK}$ and NF- $\mathrm{kB}$ in $661 \mathrm{~W}$ cells (Figure 5A-C). Furthermore, blue LED light-induced upregulation of LC3 or conversion from LC3-I to LC3-II and the formation of autophagosomes induced by autophagy were inhibited by treatment with B-ext, L-ext, and NAC (Figure 5D-F).

\section{Inhibitory effects of B-ext and L-ext on the activation of caspase-3/7 in photoreceptor cells after blue light exposure}

We analyzed caspase-3/7 activation using a caspase-3/7 assay kit after $12 \mathrm{~h}$ of blue LED light exposure. We measured the luminescence and fluorescence of cells cultured in a 96-well plate using a spectrophotometer and then calculated the caspase-3/7 activity per cell. Caspase-3/7 activity was increased by blue LED light exposure, and treatments with $10 \mu \mathrm{g} / \mathrm{mL}$ B-ext, $10 \mu \mathrm{g} /$ $\mathrm{mL}$ L-ext, and $1 \mathrm{mM}$ NAC significantly inhibited the activation of caspase-3/7 (Figure 6). 
$\mathbf{A}$

\section{Blue LED exposure}

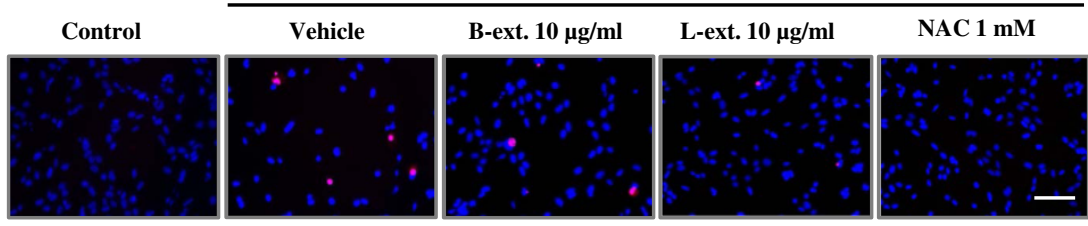

B

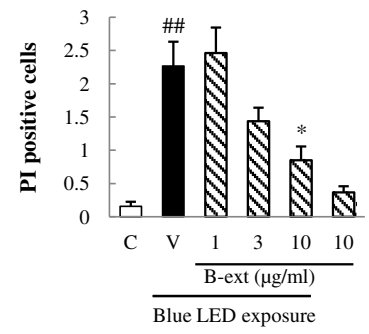

$\mathbf{E}$

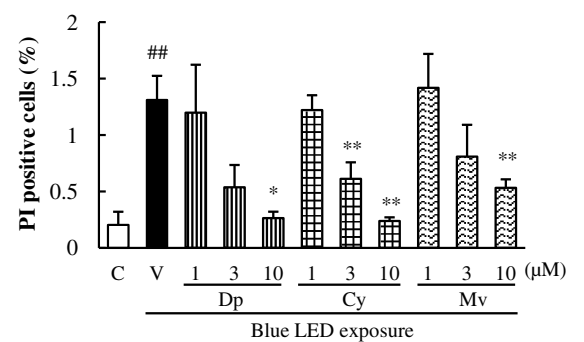

C

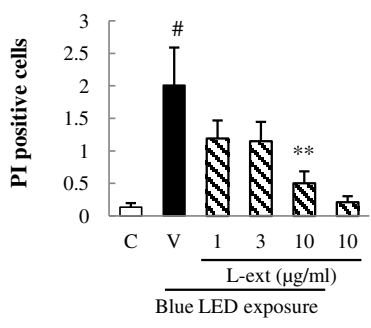

D

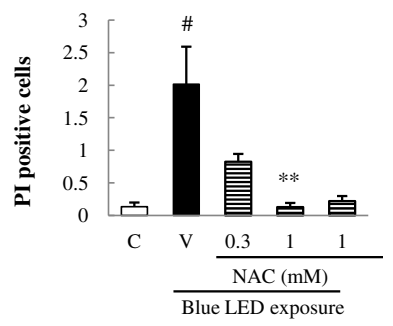

$\mathbf{F}$

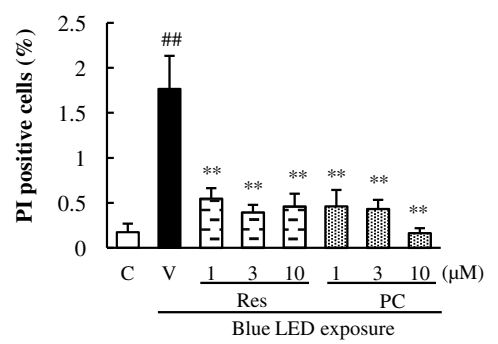

Figure 3 B-ext, L-ext, their active components, and NAC inhibited blue LED light-induced 661 W cell death. (A) Representative fluorescence microscopy images of Hoechst 33342 (blue color) and propidium iodide (PI) (red color) staining after $12 \mathrm{~h}$ of blue LED light exposure. Scale bar, $50 \mu \mathrm{m}$. Inhibitory effects of B-ext (B), L-ext (C), NAC (D), delphinidin, cyanidin, malvidin (E), resveratrol, and procyanidin B2 (F) on blue LED light-induced retinal cell death in $661 \mathrm{~W}$ cells. The number of cells exhibiting PI fluorescence was counted and expressed as a percentage of Hoechst 33342-positive cells. Cells were treated with B-ext, L-ext, NAC, delphinidin, cyanidin, malvidin, trans-resveratrol, and procyanidin B2 for $1 \mathrm{~h}$, and then exposed to $2500 \mathrm{~lx}$ of blue LED light for $6 \mathrm{~h}$. Data are means \pm SEM $(n=6)$. C, control; V, vehicle; B-ext, bilberry extract; L-ext, lingonberry extract; NAC, N-acetyl-L-cysteine; Dp, delphinidin; Cy, cyanidin; Mv, malvidin; Res, trans-resveratrol; PC, procyanidin B2. ${ }^{\# \#} p<0.01$ vs. control; ${ }^{*} p<0.05,{ }^{* *} p<0.01$ vs. the vehicle-treated group (Dunnett's multiple comparison test or Student's $t$-test).

\section{Discussion}

In this study, we focused on the protective effects of Bext, L-ext, and their active components against retinal injury caused by exposure to blue LED light (a part of the high-energy visible light) in photoreceptor cells. We first examined the effects of B-ext, L-ext, and their active components of both extracts against blue LED lightinduced photoreceptor cell damage and ROS generation. We determined the effective concentration of B-ext to be $10 \mu \mathrm{g} / \mathrm{mL}$, which contained $1.41 \mu \mathrm{g} / \mathrm{mL}(4.65 \mu \mathrm{M})$ delphinidin, $0.91 \mu \mathrm{g} / \mathrm{mL}(3.17 \mu \mathrm{M})$ cyanidin, and $0.61 \mu \mathrm{g} /$ $\mathrm{mL}(1.84 \mu \mathrm{M})$ malvidin, and that of L-ext to be $10 \mu \mathrm{g} / \mathrm{mL}$, which contained $1.06 \mu \mathrm{g} / \mathrm{mL}(4.64 \mu \mathrm{M})$ trans-resveratrol and $4.30 \mu \mathrm{g} / \mathrm{mL}(7.43 \mu \mathrm{M})$ procyanidins (Figures 2, 3 and 4 ). These findings suggest that the active components cyanidin, trans-resveratrol, and procyanidins, at concentrations of 3-10 $\mu \mathrm{M}, 3-10 \mu \mathrm{M}$, and 1-10 $\mu \mathrm{M}$, respectively, are highly effective in inhibiting blue LED light-induced cell death through ROS generation (Figures 3 and 4). B-ext contains 15 different anthocyanins, however, we could not purchase B-ext containing all anthocyanins in glycosidic form. In the results, the main anthocyanidins exerted protective effects (Figures 2, 3 and 4). We then examined cyanidin-3-glucoside, a glycosidic anthocyanin found in B-ext, and the results were similar to those obtained for cyanidin (data not shown). Thus, future studies should investigate the protective effects of other glycosidic anthocyanins in B-ext, which are absorbed into the bloodstream in the glycosylated form and metabolized in the liver and then transported as anthocyanin and anthocyanin metabolites to the eye $[21,22]$.

Blue light causes damage to mitochondrial DNA and induces free radical production in retinal cells, as 

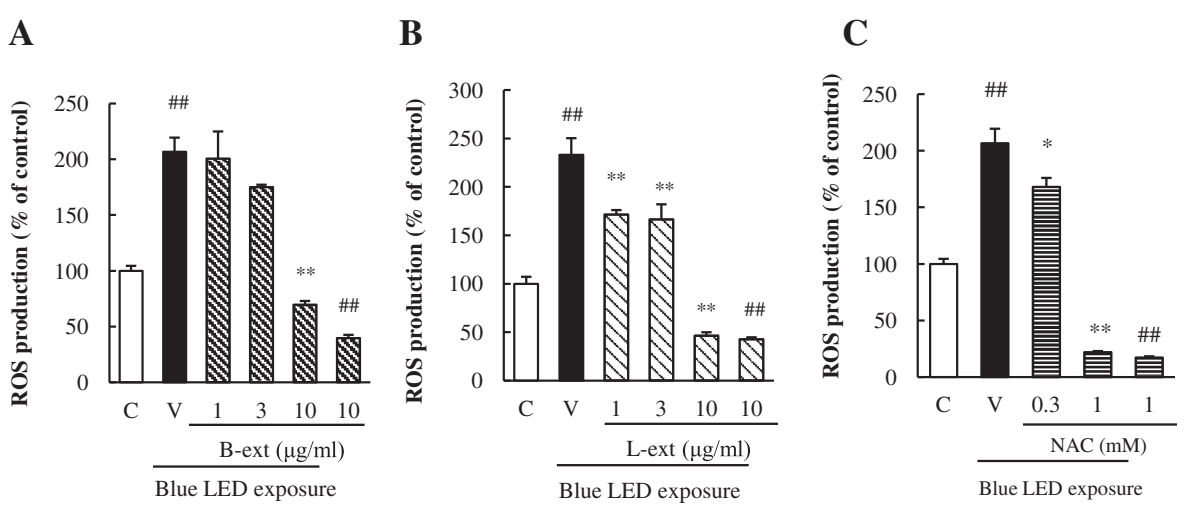

D

$\mathbf{E}$

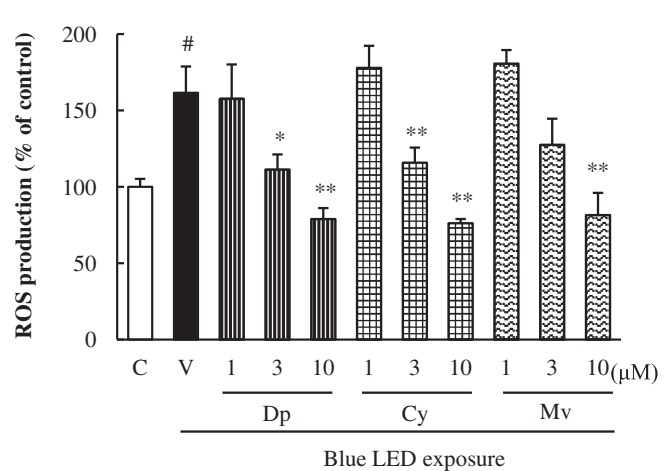

Figure 4 B-ext, L-ext, their components, and NAC inhibited production of ROS in $\mathbf{6} 61 \mathbf{W}$ cells. The cells were pretreated with B-ext, L-ext, delphinidin, cyanidin, malvidin, trans-resveratrol, and procyanidin B2 for 1 h, and then exposed to 2500 lx of blue LED light for 6 h. B-ext (A), L-ext (B), NAC (C), delphinidin, cyanidin, malvidin (D), trans-resveratrol, and procyanidin B2 (E) inhibited ROS production in cells induced by blue LED light exposure in a concentration-dependent manner. Intracellular ROS levels were determined by measuring the fluorescence of CM-H2DCFDA (excitation, $488 \mathrm{~nm}$; emission, $525 \mathrm{~nm}$ ) after blue LED light exposure for $1 \mathrm{~h}$. Data are represented as means \pm SEM $(n=6)$. C, control; $V$, vehicle; B-ext, bilberry extract; L-ext, lingonberry extract; Dp, delphinidin; Cy, cyanidin; Mv, malvidin; Res, trans-resveratrol; PC, procyanidin B2. ${ }^{\# \#} p<0.01$ vs. control; ${ }^{*} p<0.05,{ }^{* *} p<0.01$ vs. the vehicle-treated group (Dunnett's multiple comparison test or Student's t-test).

previously reported [23]. Free radicals induce lipid peroxidation, protein degeneration, and induction of apoptosis in cells. In this study, we investigated B-ext, L-ext, their active components, and NAC, and found that they exert protective effects against blue LED light-induced photoreceptor cell damage (Figures 3 and 4). We previously reported that B-ext and its anthocyanidins scavenge superoxide anion radicals and hydroxyl radicals [24]. Furthermore, trans-resveratrol and procyanidin have also been found to have free radical-scavenging activity [25,26]. In addition, anthocyanins, procyanidin B2, and trans-resveratrol enhance the antioxidant properties of intracellular glutathione and endogenous superoxide dismutase [27-29]. Thus, as shown in this study, B-ext and Lext may not only scavenge ROS but also improve the cellular ROS scavenging activity in retinal photoreceptor cells.
B-ext and L-ext containing the active components exert not only antioxidant effects but also inhibitory effects against stress response proteins induced by blue LED light exposure. p38 MAPK participates in cellular responses to mitogenic stimuli, including oxidative stress, UV exposure, and light exposure, during cell differentiation and apoptosis. The activation of p38 MAPK by light exposure induces the apoptotic transcription factor activator protein-1 (AP-1) in $661 \mathrm{~W}$ cells [30]. A previous report suggested that activation of p38 MAPK occurs because of the generation of singlet oxygen by blue light in the retina [31]. Anthocyanins, trans-resveratrol, and procyanidin B2 have antioxidant effects against ROS involving singlet oxygen [32-34]; however, NAC reacts with hydrogen peroxide, hydroxyl radical, and superoxide anion radical, but not singlet oxygen [35]. Kwon et al. [36] and Lim et al. [37] have proposed that delphinidin and cyanidin inhibit phosphorylation of MKK4 and MAPK kinase activation 

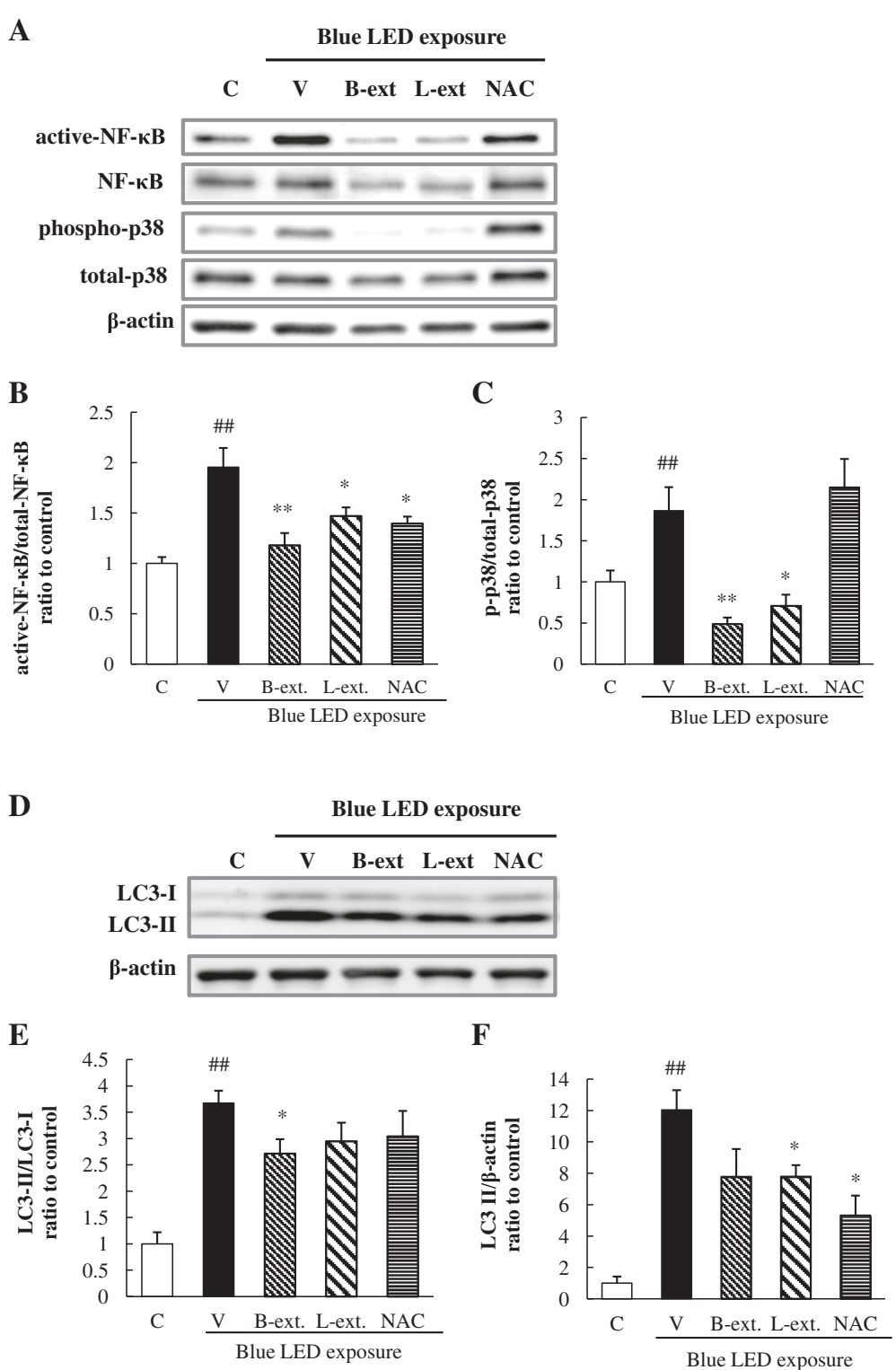

Figure 5 The effects of B-ext, L-ext, and NAC on Western blot analysis. Western blots showing the effects of B-ext, L-ext, and NAC on blue LED light-induced activation of NF-KB, phosphorylation of P38 MAPK, and conversion of LC3-I to LC3-II in 661 W cells. Cells were pretreated with B-ext, L-ext, or NAC for $1 \mathrm{~h}$, and then exposed to $2500 \mathrm{~lx}$ of blue LED light for $3 \mathrm{~h}$. Then, the cells were collected, and lysates were analyzed by western blotting. (A) Representative band images show immunoreactivities against activated NF-KB, NF-KB, phospho-p38, and total p38. (B and C) Quantitative analysis of band densities. (D) Representative band images show immunoreactivities against LC3-I and LC3-II. (E and F) Quantitative analysis of band densities. Data are represented as means \pm SEM $(n=5-6)$. C, control; $V$, vehicle; B-ext, bilberry extract; L-ext, lingonberry extract. ${ }^{\# \#} p<0.01$ vs. control; ${ }^{*} p<0.05,{ }^{* *} p<0.01$ vs. the vehicle-treated group (Student's $t$-test).

by binding to MKK4 in an ATP-competitive manner. Thus, B-ext containing delphinidin and cyanidin might also directly inhibit the blue LED light-stimulated activation of p38 MAPK.

Light exposure causes oxidative stress through NF- $\mathrm{kB}$ activation, which is related to inflammation, cancer, and cell apoptosis [38,39]. A previous in vivo study suggested that NF- $\mathrm{kB}$ colocalizes with TUNEL-positive cells in mouse retinal photoreceptors after light stimulation and causes light-induced retinal photoreceptor degeneration via the NF-kB/caspase pathway [40]. On the other hand, autophagy, or type II programmed cell death, involves the degradation of long-lived proteins in cells [41]. A previous report showed that oxidative stress and light irradiation stimulate autophagy in photoreceptor cells [42]; in addition, 3-methyladenine, an inhibitor of autophagy, 


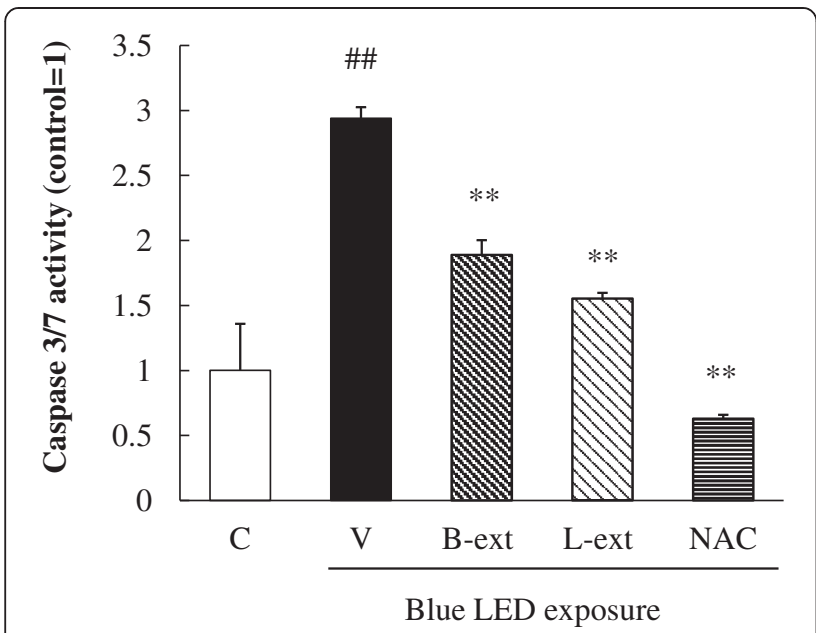

Figure 6 Effects of B-ext, L-ext, and NAC on caspase 3/7 activation in $\mathbf{6 6 1} \mathrm{W}$ cells. Cells were pretreated with B-ext, L-ext, or NAC for $1 \mathrm{~h}$, and then exposed to $2500 \mathrm{~lx}$ of blue LED light for $6 \mathrm{~h}$. The luminescence and fluorescence of the cells cultured in a 96-well plate were measured with a spectrophotometer, and the caspase-3/ 7 activity per cell was calculated. Data are represented as means \pm SEM $(n=6)$. C, control; V, vehicle; B-ext, bilberry extract; L-ext, lingonberry extract. ${ }^{\# \#} p<0.01$ vs. control; ${ }^{* *} p<0.01$ vs. the vehicle-treated group (Student's $t$-test).

prevents photoreceptor cell death induced by activated caspase- 3 with $\mathrm{H}_{2} \mathrm{O}_{2}$ treatment [42]. In this study, the large amount of ROS generated by blue LED light stimulation and the subsequent autophagy activation in photoreceptor cells might contribute, at least in part, to the blue LED light-induced photoreceptor cell death. Furthermore, a previous report suggested that activated caspase- $3,-7$, and -8 play a role as pro-autophagic agents [43]. Caspase-3/7 play an essential role in photoreceptor cell apoptosis [7] and are activated by stimulation of oxidative stress, endoplasmic reticulum stress [44], and p38 MAPK phosphorylation [45]. In the present study, B-ext and L-ext containing polyphenols might inhibit the activation of NF- $\mathrm{kB}$, autophagy (as the upregulation of LC3-II), and caspase-3/7 mainly through suppression of ROS generation induced by blue LED light exposure (Figures 5 and 6). In addition, NAC might inhibit the activation of caspase-3/7 inducing cell death through scavenging ROS except for singlet oxygen. Finally, we investigated the effects of combination with both B-ext and L-ext. Although we found additive effects of both extracts (data not shown), the difference of action mechanisms in between B-ext and L-ext was not shown except for inhibiting ROS generation.

The metabolism of orally administered anthocyanins, resveratrol, and procyanidins in animals and humans has been reported previously. In a previous human study, plasma concentrations of anthocyanins ranged between
0.56 and $4.46 \mathrm{nmol} / \mathrm{L}$ after consumption of cranberry juice containing $94.47 \mathrm{mg}$ of anthocyanins in 15 participants [21]. On the other hand, in a previous study using pigs orally administered with blueberry powder, anthocyanins have been detected in the liver, brain, and eyes [46]. Another previous in vivo study using murine ocular inflammation model demonstrated that oral administration of B-ext at $500 \mathrm{mg} / \mathrm{kg}$ body weight for $4 \mathrm{~d}$ prevented inflammatory retinal damage and visual function in mice [14]. Although the plasma concentration of anthocyanins after oral administration may be lower than the effective concentrations in vitro in the present study, anthocyanins may be able to reach the ocular tissues and may have potential eye health benefits. Several studies have shown the biokinetics of resveratrol and procyanidin in humans $[47,48]$, and the plasma concentrations were approximately the effective doses of both resveratrol and procyanidin used in our study. In further research, to determine the metabolism of those components in the eye and investigate the protective effects against blue LED light-induced photoreceptor damage in vivo would be necessary in the case of B-ext, L-ext, and those active components.

\section{Conclusion}

In conclusion, we have demonstrated that bilberry and lingonberry containing anthocyanidins, procyanidin, and resveratrol exert protective effects against blue LED light-induced retinal photoreceptor cell damage by regulating the activation of NF-kB, p38 MAPK, autophagy, and caspase-3/7 mainly through suppression of ROS generation.

\section{Abbreviations}

B-ext: Bilberry extract; CM-H 2 DCFDA: 5-(and )-chloromethyl-2,7-

dichlorodihydrofluorescein diacetate acetyl ester;

DCFH: Dichlorodihydrofluorescein; JNK: c-Jun N-terminal kinases;

LED: L-ext lingonberry extract; MAPK: Mitogen-activated protein kinase; NAC: N-Acetyl-L-cysteine; NF-KB: Nuclear factor-kappa B; PI: Propidium iodide; ROS: Reactive oxygen species; WST-8: 2-(2-methoxy-4-nitrophenyl)- 3(4-nitrophenyl)-5-(2,4-disulfophenyl)-2H-tetrazolium monosodium salt.

\section{Competing interests}

The authors declared that they have no competing interests.

\section{Authors' contributions}

$\mathrm{KO}$ designed the study, performed the tests, analyzed the data obtained and drafted this paper. YK and KT supported for design of the study and performance of the tests. SK provided the bilberry and lingonberry extracts. MS and HH supervised the execution of the study. All authors contributed to the manuscript preparation and approved of the final paper.

\section{Author details}

'Molecular Pharmacology, Department of Biofunctional Evaluation, Gifu Pharmaceutical University, 1-25-4 Daigaku-nishi, Gifu 501-1196, Japan.

${ }^{2}$ Wakasa Seikatsu Co. Ltd., 22 Naginataboko-cho, Shijo-Karasuma,

Shimogyo-ku, Kyoto 600-8008, Japan.

Received: 27 January 2014 Accepted: 27 March 2014

Published: 2 April 2014 


\section{References}

1. Glazer-Hockstein C, Dunaief JL: Could blue light-blocking lenses decrease the risk of age-related macular degeneration? Retina 2006, 26:1-4.

2. Margrain TH, Boulton M, Marshall J, Sliney DH: Do blue light filters confer protection against age-related macular degeneration? Prog Retin Eye Res 2004, 23:523-531.

3. van Norren D, Schellekens P: Blue light hazard in rat. Vis Res 1990, 30:1517-1520

4. Koide R, Ueda TN, Dawson WW, Hope GM, Ellis A, Somuelson D, Ueda T, Iwabuchi S, Fukuda S, Matsuishi M, Yasuhara H, Ozawa T, Armstrong D: [Retinal hazard from blue light emitting diode]. Nippon Ganka Gakkai zasshi 2001, 105:687-695.

5. Sparrow JR, Zhou J, Ben-Shabat S, Vollmer H, Itagaki Y, Nakanishi K: Involvement of oxidative mechanisms in blue-light-induced damage to A2E-laden RPE. Invest Ophthalmol Vis Sci 2002, 43:1222-1227.

6. Sparrow JR, Cai B: Blue light-induced apoptosis of A2E-containing RPE: involvement of caspase-3 and protection by Bcl-2. Invest Ophthalmol Vis Sci 2001, 42:1356-1362.

7. Wu J, Gorman A, Zhou X, Sandra C, Chen E: Involvement of caspase-3 in photoreceptor cell apoptosis induced by in vivo blue light exposure. Invest Ophthalmol Vis Sci 2002, 43:3349-3354.

8. Grimm C, Wenzel A, Williams T, Rol P, Hafezi F, Reme C: Rhodopsinmediated blue-light damage to the rat retina: effect of photoreversal of bleaching. Invest Ophthalmol Vis Sci 2001, 42:497-505.

9. Morazzoni PBE: Vaccinium myrtillus. Fitoterapia 1996, 67:3-29.

10. Morazoni PMMJ: Activity of myrtocyan ${ }^{\oplus}$, an anthocyanoside complex from Vaccinium myrtillus (VMA), on platelet aggregation and adhesiveness. Fitoterapia 1990, 61:13-21.

11. Lietti A, Cristoni A, Picci M: Studies on Vaccinium myrtillus anthocyanosides, I. Vasoprotective and antiinflammatory activity. Arzneimittelforschung 1976, 26:829-832.

12. Colantuoni A, Bertuglia S, Magistretti MJ, Donato L: Effects of Vaccinium Myrtillus anthocyanosides on arterial vasomotion. Arzneimittelforschung 1991, 41:905-909.

13. Bastide P, Rouher F, Tronche P: Rhodopsin and anthocyanosides. Apropos of various experimental facts. Bull Soc Ophtalmol Fr 1968, 68:801-807.

14. Miyake S, Takahashi N, Sasaki M, Kobayashi S, Tsubota K, Ozawa Y: Vision preservation during retinal inflammation by anthocyanin-rich bilberry extract: cellular and molecular mechanism. Lab Investig 2012, 92:102-109.

15. Fursova A, Gesarevich OG, Gonchar AM, Trofimova NA, Kolosova NG: Dietary supplementation with bilberry extract prevents macular degeneration and cataracts in senesce-accelerated OXYS rats. Adv Gerontol 2005, 16:76-79.

16. Matsunaga N, Imai S, Inokuchi Y, Shimazawa M, Yokota S, Araki Y, Hara H: Bilberry and its main constituents have neuroprotective effects against retinal neuronal damage in vitro and in vivo. Mol Nutr Food Res 2009, 53:869-877

17. Matsunaga N, Chikaraishi Y, Shimazawa M, Yokota S, Hara H: Vaccinium myrtillus (bilberry) extracts reduce angiogenesis in vitro and in vivo. Evid Based Complement Alternat Med 2010, 7:47-56

18. Leduc C, Coonishish J, Haddad P, Cuerrier A: Plants used by the Cree nation of eeyou istchee (Quebec, Canada) for the treatment of diabetes: a novel approach in quantitative ethnobotany. J Ethnopharmacol 2006 105:55-63.

19. Ogawa K, Tsuruma K, Tanaka J, Kakino M, Kobayashi S, Shimazawa M, Hara $\mathrm{H}$ : The protective effects of bilberry and lingonberry extracts against UV light-induced retinal photoreceptor cell damage in vitro. J Agric Food Chem 2013, 61:8.

20. Tanaka J, Nakanishi T, Ogawa K, Tsuruma K, Shimazawa M, Shimoda H, Hara $\mathrm{H}$ : Purple rice extract and anthocyanidins of the constituents protect against light-induced retinal damage in vitro and in vivo. J Agric Food Chem 2011, 59:528-536

21. Milbury PE, Vita JA, Blumberg JB: Anthocyanins are bioavailable in humans following an acute dose of cranberry juice. J Nutr 2010, 140:1099-1104.

22. Matsumoto H, Nakamura Y, lida H, Ito K, Ohquro H: Comparative assessment of distribution of blackcurrant anthocyanins in rabbit and rat ocular tissues. Exp Eye Res 2006, 83:348-356.

23. Godley BF, Shamsi FA, Liang FQ, Jarrett SG, Davies S, Boulton M: Blue light induces mitochondrial DNA damage and free radical production in epithelial cells. J Biol Chem 2005, 280:21061-21066.
24. Ogawa K, Oyagi A, Tanaka J, Kobayashi S, Hara H: The protective effect and action mechanism of Vaccinium myrtillus $L$. on gastric ulcer in mice. Phytother Res 2011, 25:1160-1165.

25. Leonard SS, Xia C, Jiang BH, Stinefelt B, Klandorf H, Harris GK, Shi X: Resveratrol scavenges reactive oxygen species and effects radical-induced cellular responses. Biochem Biophys Res Commun 2003, 309:1017-1026.

26. Jorge M, Ricardo Da Silva ND, Yvette F, Salvador M: Oxygen free radical scavenger capacity in aqueous models of different procyanidins from grape seeds. J Agric Food Chem 1991, 39:1549-1552.

27. Pintea A, Rugina D, Pop R, Bunea A, Socaciu C, Diehl HA: Antioxidant effect of trans-resveratrol in cultured human retinal pigment epithelial cells. J Ocul Pharmacol Ther 2011, 27:315-321.

28. Durukan AH, Evereklioglu C, Hurmeric V, Kerimoglu H, Erdurman C, Bayraktar MZ, Mumcuoglu T: Ingestion of $\mathrm{IH} 636$ grape seed proanthocyanidin extract to prevent selenite-induced oxidative stress in experimental cataract. J Cataract Refract Surg 2006, 32:1041-1045.

29. Hildesheim J, Awwad RT, Fornace AJ Jr: p38 Mitogen-activated protein kinase inhibitor protects the epidermis against the acute damaging effects of ultraviolet irradiation by blocking apoptosis and inflammatory responses. J Invest Dermatol 2004, 122:497-502.

30. Yang LP, Zhu XA, Tso MO: Role of NF-kappaB and MAPKs in light-induced photoreceptor apoptosis. Invest Ophthalmol Vis Sci 2007, 48:4766-4776.

31. Rozanowska M, Wessels J, Boulton M, Burke JM, Rodgers MA, Truscott TG, Sarna T: Blue light-induced singlet oxygen generation by retinal lipofuscin in non-polar media. Free Radic Biol Med 1998, 24:1107-1112.

32. De Rosso W, Moran Vieyra FE, Mercadante AZ, Borsarelli CD: Singlet oxygen quenching by anthocyanin's flavylium cations. Free Radic Res 2008, 42:885-891.

33. Olas B, Zbikowska HM, Wachowicz B, Krajewski T, Buczynski A, Magnuszewska A: Inhibitory effect of resveratrol on free radical generation in blood platelets. Acta Biochim Pol 1999, 46:961-966

34. Ariga $\mathrm{T}$ : The antioxidative function, preventive action on disease and utilization of proanthocyanidins. Biofactors 2004, 21:197-201.

35. Aruoma Ol, Halliwell B, Hoey BM, Butler J: The antioxidant action of $\mathrm{N}$-acetylcysteine: its reaction with hydrogen peroxide, hydroxyl radical, superoxide, and hypochlorous acid. Free Radic Biol Med 1989, 6:593-597.

36. Kwon JY, Lee KW, Kim JE, Jung SK, Kang NJ, Hwang MK, Heo YS, Bode AM, Dong Z, Lee HJ: Delphinidin suppresses ultraviolet B-induced cyclooxygenases-2 expression through inhibition of MAPKK4 and PI-3 kinase. Carcinogenesis 2009, 30:1932-1940.

37. Lim TG, Kwon JY, Kim J, Song NR, Lee KM, Heo YS, Lee HJ, Lee KW: Cyanidin-3-glucoside suppresses B[a]PDE-induced cyclooxygenase-2 expression by directly inhibiting Fyn kinase activity. Biochem Pharmacol 2011, 82:167-174.

38. Gloire G, Legrand-Poels S, Piette J: NF-kappaB activation by reactive oxygen species: fifteen years later. Biochem Pharmacol 2006, 72:1493-1505.

39. Doyle SL, O'Neill LA: Toll-like receptors: from the discovery of NFkappaB to new insights into transcriptional regulations in innate immunity. Biochem Pharmacol 2006, 72:1102-1113.

40. Wu T, Chiang SK, Chau FY, Tso MO: Light-induced photoreceptor degeneration may involve the NF kappa B/caspase-1 pathway in vivo. Brain Res 2003, 967:19-26.

41. Nixon RA: The role of autophagy in neurodegenerative disease. Nat Med 2013, 19:983-997.

42. Kunchithapautham K, Rohrer B: Apoptosis and autophagy in photoreceptors exposed to oxidative stress. Autophagy 2007, 3:433-441.

43. Kang R, Zeh HJ, Lotze MT, Tang D: The Beclin 1 network regulates autophagy and apoptosis. Cell Death Differ 2011, 18:571-580.

44. Tsuruma K, Shimazaki H, Nakashima K, Yamauchi M, Sugitani S, Shimazawa $\mathrm{M}$, linuma $\mathrm{M}$, Hara $\mathrm{H}$ : Annatto prevents retinal degeneration induced by endoplasmic reticulum stress in vitro and in vivo. Mol Nutr Food Res 2012, $56: 713-724$.

45. Zhuang S, Demirs JT, Kochevar IE: p38 mitogen-activated protein kinase mediates bid cleavage, mitochondrial dysfunction, and caspase-3 activation during apoptosis induced by singlet oxygen but not by hydrogen peroxide. J Biol Chem 2000, 275:25939-25948.

46. Kalt W, Blumberg JB, McDonald JE, Vinqvist-Tymchuk MR, Fillmore SA, Graf BA, O'Leary JM, Milbury PE: Identification of anthocyanins in the liver, eye, and brain of blueberry-fed pigs. J Agric Food Chem 2008, 56:705-712.

47. Boocock DJ, Faust GE, Patel KR, Schinas AM, Brown VA, Ducharme MP, Booth TD, Crowell JA, Perloff M, Gescher AJ, Steward WP, Brenner DE: Phase 
I dose escalation pharmacokinetic study in healthy volunteers of resveratrol, a potential cancer chemopreventive agent. Cancer Epidemiol Biomarkers Prev 2007, 16:1246-1252.

48. Holt RR, Lazarus SA, Sullards MC, Zhu QY, Schramm DD, Hammerstone JF, Fraga CG, Schmitz HH, Keen CL: Procyanidin dimer B2 [epicatechin(4beta-8)-epicatechin] in human plasma after the consumption of a flavanol-rich cocoa. Am J Clin Nutr 2002, 76:798-804.

doi:10.1186/1472-6882-14-120

Cite this article as: Ogawa et al.: Protective effects of bilberry and

lingonberry extracts against blue light-emitting diode light-induced retinal photoreceptor cell damage in vitro. BMC Complementary and Alternative Medicine 2014 14:120.

\section{Submit your next manuscript to BioMed Central and take full advantage of:}

- Convenient online submission

- Thorough peer review

- No space constraints or color figure charges

- Immediate publication on acceptance

- Inclusion in PubMed, CAS, Scopus and Google Scholar

- Research which is freely available for redistribution 